\section{Prevalência e fatores associados à violência entre parceiros íntimos: um estudo de base populacional em Lages, Santa Catarina, Brasil, 2007}

\author{
Prevalence of intimate partner violence and \\ associated factors: a population-based study \\ in Lages, Santa Catarina State, Brazil, 2007
}

Adriana Jaqueline Anacleto 1

Kathie Njaine 1,2

Giana Zarbato Longo 1

Antonio Fernando Boing 1

Karen Glazer Peres 3

\section{Introdução}

The aim of this study was to estimate the prevalence of intimate partner violence and associated factors in Lages, Santa Catarina State, Brazil. A population-based household study included 20-59-year-old women ( $n=1,042)$ living in the urban area. The Conflict Tactics Scales - Form $\mathrm{R}$ was used to investigate verbal aggression, minor physical violence, and severe physical violence. A questionnaire covering socioeconomic and demographic variables was applied. Pearson qui-square and linear trend test were used to test associations. Prevalence rates for verbal aggression and minor and severe physical abuse within couples were $79.0 \%, 14.9 \%$, and 9.3\%, respectively. Couples under 30 years of age, with per capita income less than half the minimum wage (approximately U\$90/month), and in households with more than two family members per bedroom were more likely to report all types of violence as compared to older couples, those with better incomes, and those living with less crowding, respectively. Preventive programs and qualitative studies could be effective strategies to shed further light on intimate partner violence.

Domestic Violence; Aggression; Socioeconomic Factors
A violência entre parceiros íntimos, entendida como qualquer comportamento numa relação íntima que cause danos físicos, psicológicos ou sexuais para aqueles na relação 1 , configura-se como um dos grandes desafios na área de direitos humanos e políticas públicas. As agressões praticadas, sobretudo por homens contra as suas companheiras, impactam gravemente a qualidade de vida e a saúde das vítimas ${ }^{2,3}$. Maior prevalência de dor crônica, estresse pós-traumático, agravos por causas externas, problemas gastrintestinais e de socialização e sintomas depressivos são reportados entre pessoas que já sofreram violência pelo parceiro íntimo em relação àquelas que nunca foram vítimas dessa situação 4,5,6.

A violência é um evento interativo 7,8 , envolvendo tanto homens quanto mulheres enquanto vítimas ou autores. Porém, a freqüência com que as mulheres buscam os serviços de atenção à saúde em decorrência de graves e reiteradas agressões sofridas por seus companheiros demonstra que esse problema constitui-se em um tipo de violência de gênero que desafia a área de saúde pública em todo o mundo.

Estudo coordenado pela Organização Mundial da Saúde e conduzido em diferentes países indicou que, na maior parte das regiões investigadas, a prevalência de mulheres que sofreram violência física perpetrada pelo seu companheiro variou entre $23 \%$ e $49 \%$ 9. Quanto à violência 
sexual, os valores concentraram-se na faixa entre $10 \%$ e $50 \%$, além do que $20 \%$ a $75 \%$ das mulheres reportaram terem sofrido atos de abuso emocional ${ }^{9}$. Pesquisas indicam, ainda, que geralmente comungam-se diferentes modalidades de violência, sendo a pessoa vítima de agressão física concomitantemente ao abuso psicológico e sexual 3,10. Apesar desse ser um fenômeno global, na literatura, têm sido apontados diversos fatores associados à violência de gênero, como baixa renda, dependência financeira, histórico de violência familiar na infância e na adolescência, baixa escolaridade, uso de álcool e drogas, baixa auto-estima, fraco vínculo afetivo com a família, distúrbios de personalidade e ausência de uma rede de prevenção e proteção 1,10.

Schraiber et al. ${ }^{11}$, ao revisarem publicações sobre violência e saúde indexadas na base de dados SciELO, destacaram a crescente produção na área entre 1980 e 2005, sendo que esse último ano compreendeu $20 \%$ de toda a produção científica analisada. No campo da violência contra a mulher, a maior parte dos estudos teve, como referência, os serviços de saúde. Dentre os escassos estudos nacionais de base populacional, destaca-se a importante variação regional na prevalência de agressões entre parceiros e também entre os tipos de agressões praticadas 3,7,12. Quanto às pesquisas com homens na perspectiva de gênero, Schraiber et al. 13 relataram o seu surgimento apenas no final do período investigado (1980-2005). Essas pesquisas buscaram não se fixar no estereótipo do homem como eterno agressor e a mulher como vítima ${ }^{13}$. Esse enfoque é ainda recente e raro na literatura que descreve a prevalência de agressões no âmbito conjugal cometidas por ambos os sexos, o que torna oportuno o desenvolvimento de novas pesquisas. Estudos sobre violência de gênero em municípios de pequeno e médio porte são ainda mais escassos, limitando-se àqueles de cunho qualitativo e que demonstraram forte influência do modo de vida da região sobre a construção das relações desiguais de gênero 14,15. Fatores como a organização dos serviços de saúde, as dinâmicas populacionais e os aspectos culturais nesses municípios diferem dos encontrados em conurbações de grande porte. Assim, pesquisas nessas localidades são essenciais a fim de ampliarem conhecimentos que possam subsidiar políticas públicas mais adequadas à realidade local.

Os objetivos deste estudo foram estimar a prevalência da violência entre parceiros íntimos e identificar os fatores associados em um município de médio porte da Região Sul do país - Lages, Santa Catarina - em 2007.

\section{Métodos}

\section{Desenho e população de estudo}

Realizou-se um estudo transversal de base populacional, cuja amostra foi composta por mulheres adultas de 20 a 59 anos, residentes na zona urbana de Lages. Essa faixa etária representava, em 2006, 53,13\% da população feminina da zona urbana do município 16. Para o cálculo do tamanho da amostra, foram considerados os seguintes parâmetros: população de mulheres adultas igual a 87.405 , prevalência do desfecho (violência entre parceiros íntimos) desconhecida igual a $50 \%$ e erro amostral de 5 pontos percentuais, perfazendo uma amostra igual a 382 mulheres. Adicionou-se um efeito de delineamento igual a 2 , ao qual foram somados $20 \%$ referentes a possíveis perdas ou recusas e mais $15 \%$ para o controle dos fatores de confusão, obtendo-se uma amostra final 1.054 mulheres.

O processo de amostragem foi realizado por meio de conglomerados, isto é, primeiro, os setores censitários foram sorteados e, em seguida, os domicílios. Todas as mulheres residentes nos domicílios sorteados e pertencentes à faixa etária de interesse do estudo foram consideradas potenciais participantes da pesquisa. Com base nas informações do Instituto Brasileiro de Geografia e Estatística (IBGE) 16, foram selecionados, sistematicamente, 60 setores censitários dentre os 186 existentes em Lages e cerca de 30 domicílios por setor. As residências selecionadas foram visitadas de maio a outubro de 2007. Foram considerados como perdas os indivíduos cujos domicílios foram visitados no mínimo quatro vezes, incluídas, pelo menos, uma visita em finais de semana e outra noturna, sem que o examinador/entrevistador conseguisse localizar a pessoa a ser entrevistada ou caso houvesse recusa em participar.

\section{Variáveis e instrumentos de aferição}

Foram investigadas, como variáveis exploratórias, a renda mensal per capita em salários mínimos analisada em quartis (1,59-19,74; 0,89-1,58; 0,51-0,88 e 0,026-0,50), a escolaridade em anos de estudo $(<4$; 4-8; 9-11 e 12-15), a aglomeração (número de pessoas por cômodo/dormitório em quartis; $0,20-1,33 ; 1,34-1,50 ; 1,51-2,00$ e 2,01-11,00), a idade (20-29 anos, 30-39 anos, 40-49 anos e 50-59 anos) e o número de filhos (nenhum, um, dois e três ou mais).

Para investigar a violência entre parceiros íntimos, foi utilizado o instrumento Conflict Tactics Scales - Form R (CTS-1), adaptado para o Brasil por Hasselmann \& Reichenheim 17. O CTS-1 
procura mensurar como os casais resolvem seus desentendimentos e, de forma indireta, detectar situações conflituosas e violentas. O instrumento usa 18 itens no processo de pontuação, que abrange três escalas. A de argumentação é composta por três itens e refere-se ao uso de expressões moderadas; a de agressão verbal é formada por seis itens relacionados ao uso de insultos e ameaças com o intuito de ofender e agredir o outro; e a escala de agressão física, que é composta por nove itens, estima o uso da agressão física como forma de resolver os conflitos e ainda possui duas subescalas que buscam aferir a violência física menor e a grave.

Cada item da escala faz referência ao casal como autor de atos considerados violentos. Neste estudo, adotou-se estratégia semelhante à pesquisa conduzida em 15 capitais brasileiras e no Distrito Federal 7, que, por questão operacional, entrevistou somente mulheres. Assim, elas informaram suas próprias atitudes em situações de conflito e relataram, como proxis, as atitudes do parceiro.

Para estimar a violência entre o casal, considerou-se evento positivo quando a resposta foi afirmativa para, ao menos, um item da escala com um dos membros do casal. Consideraramse os 12 meses anteriores à entrevista como período de recordatório.

\section{Coleta de dados}

A equipe de campo foi formada por vinte entrevistadores (dez duplas), todos estudantes dos cursos da área de saúde da Universidade do Planalto Catarinense (UNIPLAC). Somente entrevistadores do sexo feminino foram destacados para aplicar o instrumento, e essas foram devidamente capacitadas para as entrevistas, inclusive para buscar um espaço de maior privacidade durante a pesquisa com as mulheres. Já os supervisores de campo foram os alunos do mestrado em Saúde Coletiva da referida universidade. O controle de qualidade dos dados foi efetuado pelo telefone, ao acaso, em $10 \%$ das entrevistadas. Realizou-se o pré-teste do questionário com trinta mulheres da mesma faixa etária da pesquisa em área de abrangência de uma unidade de saúde do município. O estudo piloto foi realizado em um setor censitário obtido por meio de sorteio e que não foi incluído no estudo propriamente dito.

\section{Análise dos dados}

Foi realizada dupla digitação dos dados por digitadores previamente treinados no programa Epi Info, versão 6.0 (Centers for Disease Control and Prevention, Atlanta, Estados Unidos). A con- sistência e a análise dos dados foram realizadas no pacote estatístico Stata 9 (Stata Corp., College Station, Estados Unidos). Realizou-se a análise descritiva das variáveis de interesse do estudo com relação aos desfechos. Para testar a associação entre os desfechos e as variáveis independentes, foram utilizados o tese do qui quadrado de Pearson e análise de tendência linear quando pertinente. Em todas as análises, considerou-se o efeito do desenho amostral. Além disso, ao se comparar a distribuição etária da amostra investigada com a disponibilizada pelo IBGE para o ano 2005, observaram-se diferenças, sobretudo, nas faixas etárias mais elevadas. Assim, foram calculados e incorporados pesos de tal modo que a distribuição por faixa etária da amostra coincidisse com as informações intercensitárias do IBGE. Tais ajustes foram conduzidos utilizandose o conjunto de comandos svy do Stata. A pesquisa foi aprovada pelo Comitê de Ética em Pesquisa da UNIPLAC sob protocolo $\mathrm{n}^{\mathrm{o}}$. 01/2007.

\section{Resultados}

A taxa de resposta foi igual a $98,9 \%(n=1.042)$. Observa-se, na Tabela 1, que, na amostra investigada, houve maior proporção de mulheres com 20-29 e 30-39 anos de idade. Quanto à escolaridade, a maioria $(45,9 \%)$ apresentava mais de 11 anos de estudo, e apenas $8 \%$ da amostra tinham menos de quatro anos de estudo. A renda familiar mensal média per capita foi igual a $\mathrm{R} \$ 475,72$ (desvio-padrão igual a R\$476,53) e variou entre $\mathrm{R} \$ 10,00$ e R\$ 5.000,00 (dados não apresentados). Apenas 16,5\% das mulheres não tinham filhos, sendo que a maioria apresentava três ou mais filhos $(32,5 \%)$. Quase um terço das mulheres $(30,9 \%)$ residia em domicílios cuja aglomeração era de, no máximo, 1,33 pessoa por cômodo/dormitório.

A Tabela 2 apresenta os resultados relativos às prevalências de agressão verbal e física menor e grave, perpetrada pelo casal, pela mulher ou pelo homem, separadamente. Não houve diferença estatisticamente significativa quando comparadas às prevalências de agressão verbal realizadas pelas mulheres e homens, porém, no casal, a prevalência foi significativamente maior do que no homem isoladamente (79,9\%; intervalo de $95 \%$ de confiança - IC95\%: 77,4; 82,3). Em relação à agressão física menor, a prevalência total encontrada no casal foi igual a 15,9\% (IC95\%: 13,0; 18,8), valor que não diferiu, estatisticamente, em relação à mulher quando analisada separadamente (13,7\%; IC95\%: 10,8; 16,6), porém foi significativamente maior do que a prevalência entre os homens $(9,8 \%$; IC95\%: 7,8; 11,8), segundo re- 
Descrição da amostra $(n=1.042)$, segundo variáveis de estudo. Lages, Santa Catarina, Brasil, 2007.

\begin{tabular}{|c|c|c|}
\hline Variáveis & $\mathrm{n}$ & $\%(\mathrm{IC} 95 \%) *$ \\
\hline \multicolumn{3}{|c|}{ Idade (anos) $[n=1.038]$} \\
\hline $20-29$ & 299 & $30,8(27,8 ; 33,7)$ \\
\hline $30-39$ & 242 & $31,9(29,4 ; 34,5)$ \\
\hline $40-49$ & 298 & $23,6(21,0 ; 26,2)$ \\
\hline $50-59$ & 199 & $13,7(11,4 ; 16,0)$ \\
\hline \multicolumn{3}{|c|}{ Renda mensal per capita em quartis de } \\
\hline \multicolumn{3}{|c|}{ salários mínimos [n=1.023] } \\
\hline $1,59-19,74$ & 231 & $20,8(17,2 ; 24,3)$ \\
\hline $0,89-1,58$ & 266 & $26,4(22,8 ; 30,1)$ \\
\hline $0,51-0,88$ & 261 & $25,5(22,5 ; 28,5)$ \\
\hline $0,026-0,50$ & 265 & $27,3(23,7 ; 30,9)$ \\
\hline \multicolumn{3}{|c|}{ Anos de estudo [n = 1.026] } \\
\hline $12-15$ & 463 & $45,9(42,8 ; 49,1)$ \\
\hline $9-11$ & 199 & $19,4(16,9 ; 21,9)$ \\
\hline $4-8$ & 274 & $26,6(24,3 ; 28,9)$ \\
\hline$<4$ & 90 & $8,0(6,1 ; 9,9)$ \\
\hline \multicolumn{3}{|c|}{ Número de filhos [ $n=1.039]$} \\
\hline Nenhum & 165 & $16,5(14,5 ; 18,5)$ \\
\hline 1 & 238 & $24,7(22,1 ; 27,4)$ \\
\hline 2 & 279 & $26,2(23,9 ; 28,5)$ \\
\hline 3 ou mais & 357 & $32,5(29,9 ; 35,2)$ \\
\hline \multicolumn{3}{|c|}{ Aglomeração (pessoas por cômodo/ } \\
\hline \multicolumn{3}{|c|}{ dormitório) $[\mathrm{n}=1.038]$} \\
\hline $0,20-1,33$ & 346 & $30,9(27,6 ; 34,2)$ \\
\hline $1,34-1,50$ & 219 & $21,2(18,3 ; 24,2)$ \\
\hline $1,51-2,00$ & 308 & $30,5(27,4 ; 33,6)$ \\
\hline $2,01-11,00$ & 165 & $17,3(15,3 ; 19,4)$ \\
\hline
\end{tabular}

*As proporções apresentadas estão ajustadas para efeito de delineamento.

Tabela 2

Tipos de agressão perpetrada pelo casal, pela mulher e pelo homem, segundo relato da mulher (prevalência e intervalos de $95 \%$ de confiança). Lages, Santa Catarina, Brasil, 2007.

\begin{tabular}{lcccccc}
\hline Tipo de agressão & \multicolumn{2}{c}{ Casal } & Mulher & & Homem \\
& $\mathbf{n}$ & $\mathbf{P *}$ (IC95\%) & $\mathbf{n}$ & $\mathbf{P} *($ IC95\%) & $\mathbf{n}$ & $\mathbf{P}$ (IC95\%) \\
\hline Verbal & $994 * *$ & $79,9(77,4 ; 82,3)$ & $995 * *$ & $76,8(74,0 ; 79,7)$ & $994 * *$ & $71,5(68,6 ; 74,3)$ \\
Física menor & $992 * *$ & $15,9(13,0 ; 18,8)$ & $992 * *$ & $13,7(10,8 ; 16,6)$ & $992 * *$ & $9,8(7,8 ; 11,8)$ \\
Física grave & $990 * *$ & $9,5(7,6 ; 11,4)$ & $991 * *$ & $6,8(5,1 ; 8,5)$ & $990 * *$ & $5,6(4,2 ; 7,1)$ \\
\hline
\end{tabular}

* As proporções apresentadas estão ajustadas para efeito de delineamento;

** Total de respondentes. 
lato feminino. Agressão física grave apresentou o mesmo perfil que a agressão física menor, isto é, aquela analisada no casal conjuntamente $(9,5 \%$; IC95\%: 7,$6 ; 11,4)$ foi semelhante à praticada pela mulher $(6,8 \%$; IC95\%: 5,1; 8,5), porém maior do que a protagonizada pelo homem (5,6\%; IC95\%: $4,2 ; 7,1)$.

Ao analisar as associações entre os tipos de agressão para o casal e as variáveis de interesse do estudo, verificou-se que, quanto mais jovens eram as mulheres, maior a prevalência de agressão verbal $(\mathrm{p}<0,01)$. A prevalência de agressão física menor foi maior quanto menor a idade $(\mathrm{p}=$ $0,02)$ e menor a renda familiar mensal per capita $(\mathrm{p}<0,01)$. Mulheres com escolaridade entre 9 e 11 anos de estudo apresentaram as maiores prevalências de agressão física menor $(\mathrm{p}=0,020)$. Observaram-se as maiores prevalências de agressão física grave no casal cuja mulher apresentava entre 20 e 29 anos $(11,2 \%)$ e entre aquelas com mais de 50 anos de idade (10,6\%). Quanto menor a renda familiar mensal per capita maior a prevalência de agressão física grave $(\mathrm{p}<0,01)$, o mesmo sendo observado quanto à escolaridade, em que as mulheres com 12 ou mais anos de estudo apresentaram a menor prevalência desse desfecho (4,8\%) (Tabela 3).

\section{Discussão}

O presente estudo analisou a prevalência e os fatores associados à violência entre parceiros íntimos em um município de médio porte do Sul do Brasil. Comparações com outras pesquisas devem ser feitas com cautela devido às possíveis diferenças metodológicas e os distintos padrões culturais que podem influenciar nos desfechos

Tabela 3

Tipos de agressão perpetrada pelo casal, pela mulher e pelo homem, segundo características demográficas, sócio-econômicas e de comportamento do casal. Lages, Santa Catarina, Brasil, 2007.

\begin{tabular}{|c|c|c|c|}
\hline Variáveis & $\begin{array}{l}\text { Agressão verbal } \\
\qquad \text { (casal) * }\end{array}$ & $\begin{array}{l}\text { Agressão física } \\
\text { menor (casal) * }\end{array}$ & $\begin{array}{l}\text { Agressão física } \\
\text { grave (casal) * }\end{array}$ \\
\hline Idade (anos) & $p<0,001$ & $p=0,002$ & $p=0,281$ \\
\hline $20-29$ & 84,6 & 19,3 & 11,2 \\
\hline $30-39$ & 81,0 & 18,6 & 9,6 \\
\hline $40-49$ & 75,9 & 9,5 & 6,2 \\
\hline $50-59$ & 72,9 & 12,2 & 10,6 \\
\hline \multicolumn{4}{|l|}{ Renda mensal per capita em quartis } \\
\hline de salários mínimos & $p=0,011$ & $p<0,001$ & $p<0,001$ \\
\hline $1,59-19,74$ & 75,4 & 11,0 & 4,6 \\
\hline $0,89-1,58$ & 79,1 & 11,4 & 6,4 \\
\hline $0,51-0,88$ & 78,8 & 16,0 & 11,1 \\
\hline $0,026-0,50$ & 84,8 & 23,4 & 14,4 \\
\hline Anos de estudo & $p=0,154$ & $p=0,020$ & $p<0,001$ \\
\hline $12-15$ & 77,2 & 10,3 & 4,8 \\
\hline $9-11$ & 80,4 & 22,4 & 12,6 \\
\hline $4-8$ & 84,0 & 19,7 & 12,8 \\
\hline$<4$ & 78,9 & 14,6 & 13,1 \\
\hline Número de filhos & $p=0,733$ & $p=0,517$ & $p=0,098$ \\
\hline Nenhum & 77,2 & 13,2 & 5,1 \\
\hline 1 & 80,6 & 15,1 & 9,8 \\
\hline 2 & 79,8 & 17,1 & 9,9 \\
\hline 3 ou mais & 80,7 & 16,9 & 11,0 \\
\hline Pessoas por cômodo/dormitório & $p<0,001$ & $p<0,001$ & $p=0,001$ \\
\hline $0,20-1,33$ & 73,5 & 10,5 & 4,8 \\
\hline $1,34-1,50$ & 80,7 & 15,9 & 11,6 \\
\hline $1,51-2,00$ & 81,4 & 15,1 & 9,0 \\
\hline $2,01-11,00$ & 86,9 & 26,5 & 15,6 \\
\hline
\end{tabular}

* As proporções apresentadas estão ajustadas para efeito de delineamento. 
investigados. A versão original em inglês do instrumento utilizado (CTS-1) apresenta boa confiabilidade e validade concorrente de constructo e de conteúdo ${ }^{18}$. A adaptação transcultural do CTS-1 para a língua portuguesa também se mostrou adequada e permitiu seu uso junto à população brasileira 17. Por questões operacionais, a mulher foi a respondente das violências ocorridas entre o casal. Como limitação dessa opção, pode-se supor a tendência de as entrevistadas superestimarem a violência cometida pelo parceiro e subdeclarar seus próprios atos violentos 7 . Contudo, essa tendência pode se modificar, dependendo do contexto sócio-cultural em que são construídas as relações de gênero.

A prevalência de agressão verbal global foi bastante elevada (79\%), sendo semelhante ao relatado em estudo que envolveu 15 capitais brasileiras $(78,3 \%) 7$. Neste estudo nacional, entretanto, foi observada grande variação entre as cidades investigadas, sendo a menor prevalência encontrada em Campo Grande, Mato Grosso do Sul (61,7\%), e a maior, em Belo Horizonte, Minas Gerais $(85,6 \%)$ 7. Por outro lado, a prevalência de agressão verbal total no casal observada na pesquisa em Lages foi menor do que a encontrada em Florianópolis $(82,8 \%)$ 7, capital de Santa Catarina. Em relação à agressão física menor no casal, a prevalência (14,8\%), no presente estudo, foi inferior à média das quinze capitais brasileiras $(21,5 \%)^{8}$. Na pesquisa nacional, a prevalência desse tipo de agressão foi maior em Belém, Pará $(34,7 \%)$ e menor em João Pessoa, Paraíba $(12,8 \%)$ 7. Situação semelhante ocorreu em relação à agressão física grave, em que, em Lages, foi de $9,3 \%$, e, no agregado das quinze capitais, foi de $12,9 \%$, destacando-se Belém com a maior prevalência $(22,1 \%)$ e Florianópolis $(8,2 \%)$ com a menor 7 .

Nas análises estratificadas por gênero, o presente estudo apontou que as mulheres declararam cometer tantos atos de violência quanto referiram para os homens. Esse achado é corroborado por outros estudos que indicaram prevalência similar ou maior entre as mulheres de violência perpetrada contra o parceiro 19,20 . Whitaker et al. 20, ao analisarem dados sobre violência entre parceiros íntimos na população de 18 a 28 anos nos Estados Unidos, descreveram que, em $24 \%$ dos relacionamentos, havia algum tipo de violência e que, em metade desses, os atos eram recíprocos entre homem e mulher. Nos casos de não reciprocidade, as mulheres foram as agressoras em cerca de $70 \%$ dos casos. Nesse sentido, para o melhor entendimento dos atos de violência, é preciso considerar a dinâmica dessas relações e o contexto familiar que agem em conjunto com os demais fatores individuais e estruturais envolvidos na violência 21 . Deve-se ressaltar que os estudos de base populacional parecem detectar melhor os casos de violência moderada, o que, artificialmente, poderia majorar a violência perpetrada pela mulher em relação a do homem. Além disso, ressaltam-se as poucas indicações de que a mulher cometa agressões contra o parceiro na mesma intensidade e severidade de violência dirigida a ela pelo homem 22 . Outra possível explicação para a similaridade das prevalências observadas refere-se a fatores culturais, ou seja, a subestimação das mulheres em relação à violência praticada por seus parceiros, sobretudo a psicológica, que é naturalizada e socialmente aceita, embora estudos qualitativos apontem que esse tipo de abuso é, muitas vezes, considerado pelas mulheres mais degradante do que a violência física 1 .

Nossos achados, oriundos de análises bivariadas, corroboram os de outros estudos no que se refere à maior prevalência de violência, principalmente a física menor e maior entre indivíduos com baixa escolaridade, renda mais baixas e vivendo em locais precários, cujo nível de aglomeração é maior 18. Em estudo transversal conduzido nos Estados Unidos, Breiding et al. 23 descreveram que, em relação ao grupo de renda mais elevada, mulheres do estrato de menor rendimento apresentavam 2,5 vezes mais chance de terem sido vítimas de violência perpetrada pelo parceiro. A mesma associação foi descrita quando os homens foram as vítimas, indicando quase o dobro de chance de ocorrência de violência entre os de baixa renda quando comparados aos mais privilegiados economicamente. Outros estudos epidemiológicos com delineamentos transversal 24, ecológico 25 , caso-controle 26 e de coorte 27 indicaram associação entre condição sócio-econômica mais desfavorável e maior prevalência de violência entre parceiros íntimos.

Segundo Heise 8 , a violência entre parceiros íntimos é modulada pela interação de fatores individuais, situacionais e sócio-culturais. Na proposta conceitual da autora, são descritos quatro círculos. O mais interno abrange as características individuais das pessoas (biológicas e de história pessoal); o seguinte se refere ao contexto imediato no qual ocorre a violência (a família, por exemplo); o terceiro círculo está relacionado às instituições e às estruturas sociais da comunidade (como as redes sociais); por fim, o círculo mais externo compreende o meio econômico, social e cultural. A constatação, no presente estudo, da associação entre condições socioeconômicas e violência indica que ações voltadas a minimizar a violência entre parceiros íntimos devem coadunar iniciativas em diferentes perspectivas, sendo o contexto sócio-econômico 
importante fator a pautar as políticas públicas centrais e locais.

Os dados deste estudo reforçam a idéia de que a violência é relacional 7,8 e que se apresenta sob diversas formas, dependendo do contexto sócio-cultural onde se manifesta, reforçando a importância de pesquisas além de grandes centros urbanos. Nessa perspectiva, aponta-se a necessidade de criação de serviços de atenção especializados às pessoas em situação de violência que considerem a realidade sócio-econômica e cultural local e também que englobem uma abordagem da problemática que envolva o casal, e, de forma mais ampla, a família. Atualmente, alguns serviços de saúde e de atenção às pessoas em situação de violência no país têm sido submetidos a avaliações, o que demonstra a preocupação de pesquisadores e profissionais com a questão da qualidade do atendimento, incluindo desde a identificação dos casos, dos encaminhamentos e os desafios enfrentados pelos profissionais de saúde 28 . Nesse sentido, o conhecimento da prevalência da violência entre parceiros e dos fatores que atravessam essas relações são importantes para a implantação de estratégias de atendimento nas unidades de saúde e nos serviços em geral que prestam assistência às vítimas 3,29 .

Em relação à defesa dos direitos da mulher, a criação de leis nacionais e internacionais e o papel do movimento feminista vêm garantindo dispositivos de proteção importantes. Nesse contexto, destacam-se a Lei $n^{o}$. 11.340 (Lei Maria da Penha) ${ }^{30}$, que pune a violência doméstica e familiar contra a mulher, e a criação, pelo Ministério da Saúde, de normas e políticas estratégicas em relação à violência contra a mulher. Como exemplo, pode ser citada a criação, em 2004, do Plano Nacional de Políticas para Mulheres 31, que inclui as ações de enfrentamento da violência contra as mulheres como um dos principais objetivos. No entanto, ainda há muito que se avançar no tema 13. Destaca-se, por fim, a importância da criação de grupos e movimentos voltados para reflexão e responsabilização para homens autores de violência contra mulheres. Essas iniciativas, ainda que raras, buscam oferecer um espaço aos homens para discutir suas atitudes e suas experiências, ampliar o entendimento da dinâmica relacional e evitar a reincidência da violência e a cristalização de papéis 32,33 .

No que diz respeito à temática sobre a violência entre parceiros íntimos, nosso estudo reitera a necessidade de aprofundar a compreensão sobre os fatores pessoais, situacionais, sociais e culturais e a interação entre eles, relacionados ao risco de agressão entre parceiros íntimos, assim como a implementação de medidas de intervenção, prevenção da violência e promoção da saúde 1 . Pesquisas com metodologia quanti-qualitativas poderiam contribuir neste sentido.

\section{Resumo}

Estimou-se a prevalência da violência entre parceiros intimos e os fatores associados em Lages, Santa Catarina, Brasil. Realizou-se um estudo transversal de base populacional domiciliar com mulheres de 20-59 anos ( $n=1.042)$, da zona urbana. As prevalências de agressão verbal, violência física menor e violência física grave foram estimadas pelo questionário Conflict Tactics Scales - Form R. Questões sobre aspectos sócio-econômicos e demográficos foram investigadas. As associações foram testadas pelo teste do qui-quadrado de Pearson e o de tendência linear. A prevalência de violência entre casais para agressão verbal, agressão física menor e agressão física grave foram de 79\%, 14,9\% e 9,3\%, respectivamente. Casais com menos de trinta anos, com renda per capita inferior a meio salário mínimo mensal e vivendo em locais com mais de dois indivíduos por cômodo dormitório apresentaram maiores prevalências das violências mensuradas quando comparados com casais mais velhos, com maior rendimento e menor aglomeração, respectivamente. Programas preventivos, além de estudos qualitativos, podem ser estratégias efetivas para melhor compreender a violência entre parceiros intimos.

Violência Doméstica; Agressão; Fatores Socioeconômicos 


\section{Colaboradores}

A. J. Anacleto participou da coleta de dados, da redação e realizou revisão crítica do artigo. K. Njaine e K. G. Peres contribuíram no planejamento do estudo, do treinamento dos observadores, da análise dos dados, da redação e realizaram revisão crítica do artigo. G. Z. Longo participou do planejamento do estudo, do treinamento dos observadores e realizou revisão crítica do artigo. A. F. Boing participou da análise dos dados, da redação e realizou revisão crítica do artigo.

\section{Referências}

1. Krug EG, Dahlberg LL, Mercy JA, Zwi AB, Lozano R. World report on violence and health. Geneva: World Health Organization; 2002.

2. Guimarães I. Violência faz mal à saúde. Brasília: Ministério da Saúde; 2004.

3. Schraiber LB, D'Oliveira AFPL, França Júnior I, Diniz S, Portella AP, Ludermir AB, et al. Prevalência da violência contra a mulher por parceiro íntimo em regiões do Brasil. Rev Saúde Pública 2007; 41:797-807.

4. Campbell JC. Health consequences of intimate partner violence. Lancet 2002; 359:1331-6.

5. Bonomi AE, Thompson RS, Anderson M, Reid RJ, Carrell D, Dimer JA, et al. Intimate partner violence and women's physical, mental, and social functioning. Am J Prev Med 2006; 30:458-66.

6. Ruiz-Pérez I, Plazaola-Castaño J, Del Río-Lozano M. Physical health consequences of intimate partner violence in Spanish women. Eur J Public Health 2007; 17:437-43.

7. Reichenheim ME, Moraes CL, Szklo A, Hasselman MH, Souza ER, Lozana JA, et al. The magnitude of intimate partner violence in Brazil: portraits from 15 capital cities and the Federal District. Cad Saúde Pública 2006; 22:425-37.

8. Heise L. Violence against women: an integrated, ecological framework. Violence Against Women 1998; 4:262-90.

\section{Agradecimentos}

Pesquisa financiada pelo Programa de Pós-graduação em Saúde Coletiva da Universidade do Planalto Catarinense. R. G. Peres é bolsista de produtividade do Conselho Nacional de Desenvolvimento Científico e Tecnológico.
9. World Health Organization. Who multi-contry study on women's health domestic violence against women: summary report of initial results on prevalence, health outcomes and women's responses. Geneva: World Health Organization; 2005.

10. Güezmes AG. Reconocimiento de la violencia contra la mujer como un problema de salud pública. Lima: Movimiento Flora Tristán; 2003.

11. Schraiber LB, D'Oliveira ANPL, Couto MT. Violência e saúde: estudos científicos recentes. Rev Saúde Pública 2006; 40(n Esp):112-20.

12. Venturi G, Recaman M, Oliveira S. A mulher brasileira nos espaços público e privado. São Paulo: Fundação Perseu Abramo; 2004.

13. Schraiber LB, Gomes R, Couto MT. Homens e saúde na pauta da Saúde Coletiva. Ciên Saúde Coletiva $2005 ; 10: 7-17$.

14. Bitencourt H. Vidas Marcadas: um estudo das relações de gênero na família em Lages [Dissertação de Mestrado]. Florianópolis: Universidade Federal de Santa Catarina; 2001.

15. Porto MR. Gravidez e Relações Violentas: Representação da Violência Doméstica no Município de Lages-SC [Dissertação de Mestrado]. Florianópolis: Universidade Federal de Santa Catarina; 2002.

16. Instituto Brasileiro de Geografia e Estatística. Censo demográfico de 2000. http://www.ibge.gov.br (acessado em 19/Set/2006). 
17. Halsselmann MH, Reichenheim ME. Adaptação transcultural da versão para o português da Conflict Tactics Scales Form R (CTS-1), usada para aferir violência no casal: equivalência semântica e de mensuração. Cad Saúde Pública 2003; 19:1083-93.

18. Straus MA, Gelles JR. Physical violence in american families: risk factors and adaptations to violence in 8,145 Families. New Brunswich: Transaction; 1995.

19. Moreno-Martín F. Violence in couples. Rev Panam Salud Publica 1999; 5:245-58.

20. Whitaker DJ, Haileyesus T, Swahn M, Saltzman LS. Differences in frequency of violence and reported injury between relationships with reciprocal and nonreciprocal intimate partner violence. Am J Public Health 2007; 97:941-7.

21. Castro R, Riquer F. La investigación sobre violencia contra las mujeres en América Latina: entre el empirismo ciego y la teoría sin datos. Cad Saúde Pública 2003; 19:135-46.

22. Johnson MP, Ferraro KJ. Research on domestic violence in the 1990s: making distinctions. J Marriage Fam 2000; 62:948-63.

23. Breiding MJ, Black MC, Ryan GW. Prevalence and risk factors of intimate partner violence in eighteen U.S. states/territories, 2005. Am J Prev Med 2008; 34:112-8.

24. Rickert VI, Wiemann CM, Harrykissoon SD, Berenson $\mathrm{AB}$, Kolb E. The relationship among demographics, reproductive characteristics, and intimate partner violence. Am J Obstet Gynecol 2002; 187:1002-7

25. Koenig M, Ahmed S, Hossain M, Mozumber K. Women's status and domestic violence in rural Bangladesh: individual and community-level effects. Demography 2003; 40:269-88.

26. Lewis BY. Psychosocial factors related to wife abuse. J Fam Violence 1987;2:1-10.

27. Fergusson D, Horwood J, Kershaw K, Shannon F. Factors associated with reports of wife assault in New Zealand. J Marriage Fam 1986; 48:407-12.
28. Centro Latino-americano de Estudos de Violência e Saúde Jorge Careli/Fundo das Nações Unidas para a Infância. Famílias: parceiras ou usuárias eventuais? Análise de serviços de atenção a famílias com dinâmicas de violência doméstica contra crianças e adolescentes. Rio de Janeiro: Centro Latino-americano de Estudos de Violência e Saúde Jorge Careli/Fundo das Nações Unidas para a Infância; 2004.

29. Schraiber LB, D'Oliveira AFPL, Couto MT, Hanada H, Kiss LB, Durand JG, et al. Violência contra mulheres entre usuárias de serviços públicos de saúde da Grande São Paulo. Rev Saúde Pública 2007; 41:359-67.

30. Brasil. Lei $n^{\circ}$. 11.340 de 7 de agosto de 2006. Cria mecanismos para coibir a violência doméstica e familiar contra a mulher, nos termos do $\$ 8^{\circ}$ do art. 226 da Constituição Federal, da Convenção sobre a Eliminação de Todas as Formas de Discriminação contra as Mulheres e da Convenção Interamericana para Prevenir, Punir e Erradicar a Violência contra a Mulher; dispõe sobre a criação dos Juizados de Violência Doméstica e Familiar contra a Mulher; altera o Código de Processo Penal, o Código Penal e a Lei de Execução Penal; e dá outras providências. Diário Oficial da União 2006; 8 ago.

31. Secretaria Especial de Políticas para Mulheres. Plano Nacional de Políticas para Mulheres. http:// 200.130.7.5/spmu/docs/PNPM.pdf (acessado em 12/Dez/2006)

32. Acosta F, Andrade FA, Bronz A. Metodologia: conversas homem a homem - grupo reflexivo de gênero. Rio de Janeiro: Instituto Noos; 2004.

33. Acosta F, Barker G. Homens, violência de gênero e saúde sexual e reprodutiva: um estudo sobre homens no Rio de Janeiro/Brasil. Rio de Janeiro: Instituto Noos; 2003

Recebido em 24/Mar/2008

Versão final reapresentada em 21/Out/2008

Aprovado em 29/Out/2008 